\title{
Effect of Using Perfluorocarbon Liquids on Postoperative Retinal Displacement in Vitrectomy for Treatment of Primary Rhegmatogenous Retinal Detachment: A Comparative Study
}

\author{
MOHAMED A. AZAB, M.Sc.*, MOSTAFA H. NABIH, M.D.**; AYMAN M. KHATTAB, M.D.** and \\ ISLAM A. MOHALHAL, M.D.* \\ Research Institute of Ophthalmology, Ministry of High Education, Giza* and Department of Ophthalmology, Faculty of Medicine, \\ Cairo University***, Cairo, Egypt
}

\begin{abstract}
Backdround: Perfluorocarbon liquids (PFCLs) are widely used for years as an adjuvant in vitrectomy for repair of retinal detachment. Studies have been made to find out the postoperative effects of using PFCLs on the retina. A new imaging modality, Fundus Autofluorescence (FAF) can describe retinal displacement after vitrectomy clearly, which we could not describe it before with other imaging modalities.
\end{abstract}

Aim of Study: To compare the incidence of retinal displacement after vitrectomy for repair of primary rhegmatogenous retinal detachment (RRD) with and without the use of (PFCLs) using (FAF), and correlate them with final best corrected visual acuity (BCVA) and postoperative symptoms of distortion if present.

Patients and Methods: A comparative, prospective, interventional, randomized study. Thirty eyes of thirty patients having primary rhegmatogenous retinal detachment were divided into 2 groups. Group A: 15 eyes underwent pars plana vitrectomy without the use of PFCLs and group B: 15 eyes underwent pars plana vitrectomy with the use of PFCLs. Fundus Autofluorescence and amsler grid were done following absorption of intraocular gas.

Results: Retinal displacement evident on FAF was $(53.3 \%)$ of cases in group A (without PFCL), and (40.0\%) of cases in group B (with PFCL), respectively $(p$-value $=0.464)$. PFCL use has no statistical significance in the incidence of retinal displacement. There was no statistically significant difference in the final mean BCVA between the two groups. Postoperative distortion was detected in $(85.7 \%)$ of eyes showing retinal displacement on FAF

Conclusion: Retinal displacement is common following RRD repair and the use of PFCLs does not seem to affect the incidence of displacement.

Key Words: Rhegmatogenous retinal detachment-Retinal displacement - Pars plana vitrectomy - Perfluorocarbon liquids - Fundus autofluorescence.

Correspondence to: Dr. Islam A. Mohalhal,

E-Mail dr.islamabdallah@hotmail.com

\section{Introduction}

THE selection of the surgical approach for repair of primary rhegmatogenous retinal detachment (RRD) is the most important indicator for postoperative functional and anatomical success [1]. The essential steps of any surgery depend on accurate identification, localization and treatment of all tears [2]. Currently the most two frequent surgical techniques are scleral buckling and pars plana vitrectomy (PPV), with pneumatic retinopexy being reserved for selected cases [3]. Pars plana vitrectomy has become more popular than scleral surgery in recent years, mainly for primary rhegmatogenous retinal detachment. Intra-surgery visualization and identification of retinal tears is broadly considered being superior to pre-surgery visualization and identification. Probably this is one among the main reasons for the tendency to use this technique as the first choice for repair of primary RRD. Yoshida et al., reported in a prospective and retrospective case series study that primary reattachment rates were of $75-100 \%$ with a final rate of $96-100 \%$ [4]

The well acclaimed use of perfluorocarbon liquids (PFCLs) in vitreoretinal surgery being due to their unique physical properties as they have a high specific gravity ranging from 1.76 to 2.03 , low surface tension, low viscosity and optical clarity making them an ideal intraoperative adjuvant in vitreoretinal surgery mainly to relocate and flatten the detached retina, so that improving the efficiency and safety of the surgery especially in complicated cases [5]

Fundus Autofluorescence (FAF) has been considered as a novel imaging modality to identify a 
new postoperative entity following retinal detachment surgery which is retinal displacement and various studies have been directed to correlate between it and the functional outcome of surgery in terms of visual acuity and postoperative symptoms [6]. FAF elegantly depicts this shift via lines of increased autofluorescence, which reflect the caliber and orientation of the adjacent retinal vessels. These lines indicate the original location of the retinal vessels, which have been displaced with the retina after retinal reattachment. Dell'Omo et al., named these lines as retinal vessel printings (RVPs) [7]. While Lee et al., named them as RPE vessel ghost [8]. The reason why lines of increased autofluorescence indicate the preoperative site of retinal vessels is unclear. Among the explanations of this phenomenon was that stated in a study by Shiragami et al., in which they suggested that the RPE cells under the retinal vessels were prevented from light irradiation for a long time and hence they maintained dark adaptation because they were shaded by the retinal vessels. After that, acute exposure to the excitation light, which was caused by translocation of the retinal vessels, may induce increased FAF signals [6]. On the other hand, Dell'Omo et al., proposed that a different composition and characteristics of fluorophores may exist between the RPE cells belonging to the shifted vessels' prints (normally shaded by the overlying vessels) and the neighboring cells (normally exposed to light) [7].

To date, there have been many studies about retinal displacement after vitrectomy [7,8]. However, in our study we compared the incidence of retinal displacement after vitrectomy for repair of primary rhegmatogenous retinal detachment with and without the use of PFCLs, using FAF which is a valuable simple imaging technique, and correlate them with the final best corrected visual acuity (BCVA) and postoperative symptoms of distortion if present.

\section{Matreial and Methods}

This study is a comparative prospective interventional randomized study. It included 30 eyes of 30 patients undergoing primary vitrectomy for repair of primary rhegmatogenous retinal detachment (RRD). The primary outcome of the study was evaluation of presence or absence of retinal displacement using FAF. Secondary outcomes were postoperative visual acuity and symptoms of distortion like metamorphopsia, if present. Patients were recruited from the outpatient clinic of the Research Institute of Ophthalmology in Giza from the period of June 2017 till April 2018. It was approved by the Institutional Review Board and was conducted in compliance with principles of Helsinki declaration.

We included patients with primary RRD (with proliferative vitreoretinopathy (PVR) grade A or B) assigned for primary vitrectomy. Cases were divided randomly into two groups A and B; each included 15 eyes: Group A: Underwent pars plana vitrectomy without the use of PFCLs. Group B: Underwent pars plana vitrectomy with the use of PFCLs.

We excluded cases with PVR more than grade $\mathrm{B}$, cases that had performed previous retinal detachment surgeries; pneumatic retinopexy, scleral buckle or PPV, cases with old standing retinal detachment, exudative or tractional retinal detachment, giant retinal tears, macular holes and pediatric cases.

\section{Baseline evaluation:}

Complete ocular examination was done including BCVA (with snellen chart, which was converted to $\log$ MAR for statistical analysis), intraocular pressure (IOP) using Goldmann applanation tonometry and anterior segment biomicroscopy. The retinal detachment in all cases was evaluated using slit lamp biomicroscopy using (Super Field lens; VOLK, Mentor, Ohio, USA) and indirect ophthalmoscopy determining extent and configuration of the retinal detachment, macular status, number and position of the breaks and grade of PVR.

\section{Surgical procedure:}

In Group A cases: PPV was done using 23gauge system (Constellation system. Alcon, Fort Worth, TX). Posterior vitreous detachment were induced, followed by core vitrectomy and assisted by the use of triamcinolone acetonide to stain the vitreous in order to achieve near complete vitreous removal, followed by shaving of the vitreous base assisted by scleral indentation. Shift to fluid air exchange, followed by drainage of subretinal fluid through retinal breaks. Finally, laser was applied around all marked breaks and augmented by 360 degrees barrage if the retina condition required this. This was followed by closure of the sclerotomies then by the use of two syringes one filled with gas and the other acting as a chimney replacing the air with the non expansile concentration (20\%) of the sulphur hexafluoride (SF6) gas. Periocular antibiotic steroid combination was given and the (IOP) was adjusted.

In Group B cases: The same steps applied except that after completion of the core vitrectomy step, PFCLs were infused over the optic disc to 
displace the subretinal fluid to the periphery followed by complete shaving of the vitreous base. This was followed by more injection of PFCL and then either apply the laser under PFCL or replace the PFCL by air and apply laser under air. After complete removal of PFCL, all cases were tamponaded with gas intra-operatively. Patients were advised to take the face down position immediately following the operation. All patients were prescribed topical antibiotics and steroids after the operation.

\section{Post-operative follow-up:}

Patients were followed-up on the $1{ }^{\text {st }}$ day postoperatively then after 1 week, 2 weeks, 1 month and 3 months respectively, examination included: BCVA by refraction; slit lamp examination, anterior segment biomicroscopy, IOP measurement using Goldmann applanation tonometry, dilated fundus examination with binocular slit-lamp biomicroscopy (using 90 diopters lens) and indirect ophthalmoscopy (using $20 \mathrm{D}$ lens). Symptoms of distortion were subjectively recorded using Amsler grid. Imaging (FAF) was typically performed following absorption of intraocular gas tamponade using Spectralis HRA+OCT (Heidelberg Engineering, Heidelberg, Germany). B-FAF (excitation wavelength at $488 \mathrm{~nm}$ and barrier filter at $500 \mathrm{~nm}$ ) and red-free pictures (excitation wavelength at $508 \mathrm{~nm}$ and barrier filter at $358 \mathrm{~nm}$ ) were obtained.

\section{Image analysis:}

Postoperatively, the presence of ghost vessels and their position (upward or downward) were analyzed in relation to the adjacent retinal vessels. According to the study published by Lee et al., which defined the ghost vessels as lines of increased autofluorescence running approximately parallel to adjacent retinal vessels, separated from them and closely reflecting their caliber and orientation. The presence of ghost vessels was taken as evidence of retinal displacement [8].

\section{Statistical analysis:}

Data were collected, revised, coded and entered to the Statistical Package for Social Science (IBM SPSS) version 20. The quantitative data were presented as mean, standard deviations when their distribution found parametric while qualitative data were presented as number. The comparison between two independent groups with qualitative data was done by using Chi-square test. The comparison between two independent groups with quantitative data and parametric distribution was done by using Independent $t$-test. The confidence interval was set to $95 \%$ and the margin of error accepted was set to $5 \%$. So, the $p$-value was considered significant as the following: $p>0.05$ : Nonsignificant, $p<0.05$ : Significant, $p<0.01$ : Highly significant.

\section{Results}

This study included 30 eyes of 30 patients divided into 2 groups 15 eyes each; Group A: underwent PPV without the use of PFCLs, Group B: underwent PPV with the use of PFCLs. With a mean age of $56.27 \pm 11.82$ for Group (A) patients and 49.13 \pm 13.09 for Group (B) patients. In Group (A) 9 eyes belonged to 9 females and the other 6 eyes belonged to 6 males, while in Group (B) 5 eyes belonged to 5 females and the other 10 eyes belonged to 10 males. The baseline characteristics of the studied groups are summarized in Table (1). Baseline characteristics of the two groups were homogeneous with no significant statistical difference between groups in terms of age and gender.

Table (1): Baseline characteristics of the studied groups.

\begin{tabular}{|c|c|c|c|c|}
\hline & $\begin{array}{c}\text { Group A } \\
\text { (Without PFCL) }\end{array}$ & $\begin{array}{c}\text { Group B } \\
\text { (With PFCL) }\end{array}$ & $p-$ & Sig. \\
\hline & No. $=15$ & No. $=15$ & & \\
\hline \multicolumn{5}{|l|}{ Age: } \\
\hline Mean \pm SD & $56.27 \pm 11.82$ & $49.13 \pm 13.09$ & 0.128 & NS \\
\hline Range & $30-72$ & $25-73$ & & \\
\hline \multicolumn{5}{|l|}{ Gender: } \\
\hline Females & $9(60.0 \%)$ & $5(33.3 \%)$ & 0.143 & NS \\
\hline Males & $6(40.0 \%)$ & $10(66.7 \%)$ & & \\
\hline Left & $6(40.0 \%)$ & $10(66.7 \%)$ & & \\
\hline $\begin{array}{ll}\mathrm{SD} & =\text { Stand } \\
\mathrm{NS} & =\text { Not Si } \\
\mathrm{P} & =\text { Probal } \\
\text { PFCL } & =\text { Perflu }\end{array}$ & $\begin{array}{l}\text { ard Deviation of age } 1 \\
\text { gnificant. } \\
\text { ility level. } \\
\text { orocarbon Liquids. }\end{array}$ & & & \\
\hline
\end{tabular}

The primary anatomical success (defined as successful retinal reattachment after the first surgery) was achieved in all 15 eyes of Group A patients (100\%) and in 14 of the 15 eyes of Group $\mathrm{B}$ patients $(93.3 \%)$. On the other hand, the final anatomical success (defined as successful retinal reattachment regardless the number of procedures) was achieved in all 30 eyes of the studied groups.

After retinal reattachment retinal ghost vessels indicating retinal displacement were observed in 14 of the 30 eyes $(46.6 \%$ ) (8 eyes in Group A $(53.3 \%)$ and 6 eyes in Group B (40.0\%). There was no statistically significant difference between both groups $(p$-value $=0.464)$. The direction of retinal displacement in all of the eyes of the studied groups was downwards (100\%) (Fig. 1) (Table 2). 
Table (2): FAF findings in the studied groups.

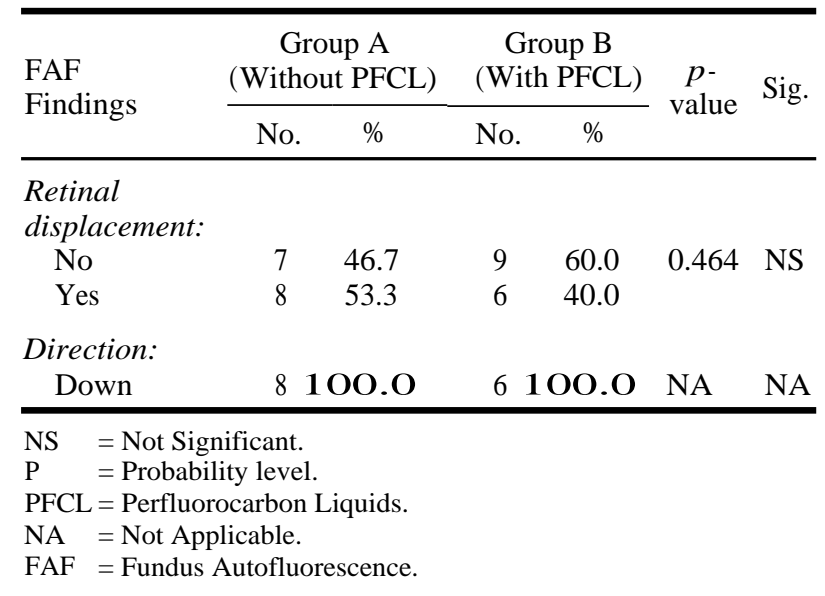

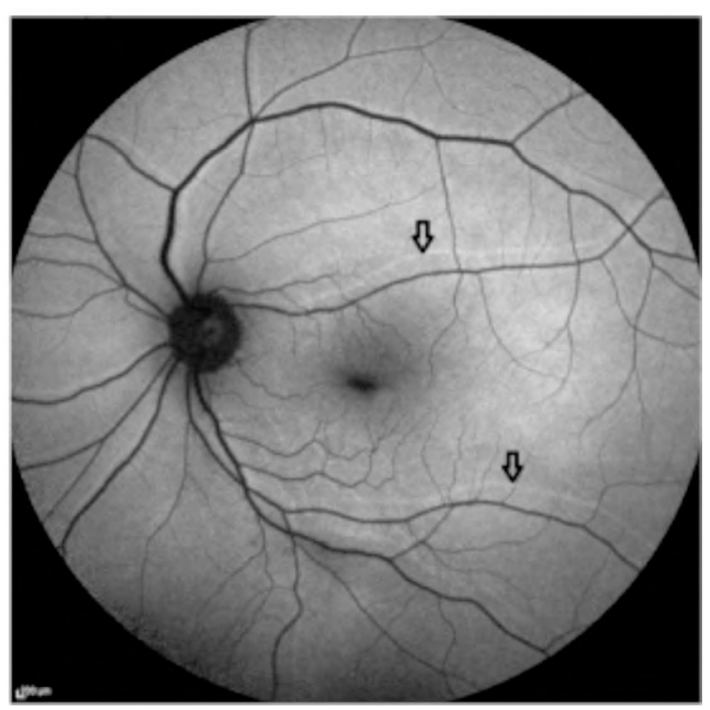

Fig. (1): Post-operative FAF of a case of retinal displacement, note the hyperfluorescent lines above \& parallel to the retinal vessels indicating downward displacement of the retina.

In Group (A) patients none of the 3 eyes having fovea sparing retinal detachments showed retinal displacement, while of the remaining 12 foveainvolving retinal detachments 8 eyes showed retinal displacement indicating that all cases $(100 \%)$ of the group (A) eyes showing retinal displacement had a fovea-involving detachment. There was no statistically significant correlation between neither the location of breaks nor the detachment extent with the incidence of retinal displacement.

Unlike Group (A), in Group (B) patients 2 eyes having fovea-sparing retinal detachment showed retinal displacement and the remaining 4 eyes had fovea-involving detachments. However similarly to Group (A) patients, there was no statistically significant correlation between neither the location of breaks nor the detachment extent with the incidence of retinal displacement.
The mean post-operative BCVA was $0.70 \pm 0.42$ for Group (A) patients and $0.85 \pm 0.42$ for Group (B) patients during the first follow-up visit following the absorption of intra-ocular gas tamponade. BCVA was measured using the Snellen's chart and converted to LogMAR for statistical analysis. There was no statistically significant difference between both groups $(p$-value $=0.327)$.

All patients underwent screening for symptoms of distortion using Amsler grid test, 9 patients from Group (A) reported to have symptoms of distortion post-operatively in comparison to the 10 patients from Group (B) who also reported to have postoperative symptoms of distortion.

In the patients with evidence of retinal displacement on FAF, symptoms of distortion were present in $85.7 \%(12 / 14)$ eyes, on the other hand in those with symptoms of distortion there was evidence of retinal displacement in $63.1 \%(12 / 19)$ eyes this may indicate that retinal displacement is not the only cause of the symptoms of distortion (Table 3).

Table (3): Correlation between Retinal displacement on FAF and Symptoms of distortion in the studied groups.

\begin{tabular}{|c|c|c|c|c|c|c|}
\hline \multirow{2}{*}{$\begin{array}{l}\text { Visual } \\
\text { Disturbances }\end{array}$} & \multicolumn{2}{|c|}{ No displacement } & \multicolumn{2}{|c|}{ Displacement } & \multirow{2}{*}{$\begin{array}{c}p- \\
\text { value }\end{array}$} & \multirow{2}{*}{ Sig. } \\
\hline & No. & $\%$ & No. & $\%$ & & \\
\hline No & 9 & 56.3 & 2 & 14.3 & & \\
\hline Metamorphopsia & 6 & 37.5 & 7 & 50.0 & & \\
\hline $\begin{array}{l}\text { Metamorphopsia+ } \\
\text { Micropsia }\end{array}$ & +1 & 6.3 & 4 & 28.6 & 0.065 & NS \\
\hline Micropsia & 0 & 0.0 & 1 & 7.1 & & \\
\hline
\end{tabular}

\section{Discussion}

PFCLs have become popular in retinal detachment surgery because of their physical properties (optical transparency, low viscosity, immiscibility with water) [9]. They allow the retina to reattach without the need for retinotomies and intraoperative stabilization of the retina in more complex situations, such as trauma with the presence of PVR.9,10 The major side effects of PFCL arise from their abnormal intraocular fate, in the vitreous cavity, subretinally or in the anterior chamber $[9,10,11]$ Benefits of the presented PFCL-free technique include, in addition to avoiding possible side effects of the lagging PFCL, better visibility of the anterior retina in the air-filled eye, which may additionally facilitate retinal reattachment [12].

In our study the primary anatomical success was achieved in all 15 eyes $(100 \%)$ of Group (A) patients where PFCL wasn't used, and in 14 of the 
15 eyes of Group (B) patients (93.3\%) where PFCL was used, which wasn't statistically significant ( $p$ value $=0.309)$, with both groups $(100 \%)$ successfully achieving the final anatomical success.

Comparable results between both approaches have been documented in literature as regards the anatomical outcomes of PPV in cases having primary RRD with primary anatomical success rates ranging from $90.0 \%$ to $98.0 \%$ in cases with routine PFCL use $[\mathbf{1 3 , 1 4 , 1 5 ]}$. It was $95.7 \%$ in the study published by Schneider et al., in which PFCL wasn't used, with all of these mentioned studies achieving a near $100 \%$ final anatomical success.

(FAF) has been reported to be an accurate mean of assessing retinal displacement, and this was the technique of choice in several studies [16]. In the study published by Lee et al., they hypothesized that the use of PFCLs might theoretically reduce the incidence of postoperative retinal displacement evident on FAF by reducing the residual postoperative SRF based on the fact that most studies attribute the displacement to the effect of the residual SRF postoperatively acting as a downward force on the retina hence causing downward displacement of the retina following retinal detachment surgery $[6,7,8]$.

In our study FAF showed retinal displacement (demonstrated by the hyperfluorescent lines running parallel to the retinal blood vessels) in 14 of the 30 eyes $(46.6 \%)$. These results are consistent with the recent study conducted by Dell'Omo et al., which documented the displacement in $41.2 \%$ of cases [7]. This was slightly less than the older studies by Shiragami et al.; Lee et al.; Cobos et al. and Codenotti et al., which were $62.8 \%, 72 \%, 60 \%$, $71.4 \%$ respectively $[\mathbf{6 , 8 , 1 6 , 1 7 ]}$

Of the 14 cases that showed retinal displacement on FAF, PFCL was used in 6 eyes $(42.8 \%)$ and not used in 8 eyes $(57.1 \%)$ hence indicating that its use has no statistical significance in the incidence of retinal displacement. This is consistent with the studies performed by Shiragami et al.; Dell'Omo et al. and Codenotti et al., who also found no statistically significant correlation between the use of PFCLs and reducing the incidence of retinal displacement $[\mathbf{6 , 7 , 1 7 ]}$

Among the 14 eyes that showed retinal displacement on FAF the direction of shift was downwards in all the eyes showing displacement This is in agreement with the studies by Shiragami et al.; Lee et al.; Codenotti et al. and Pandya et al., which all reported that retinal displacement occurs in downwards direction with gas tamponade $[6,8,17,18]$
In contrast to the study by Dell'Omo et al., which stated that displacement with gas tamponade can occur both upwards or downwards but also stating that downward displacement is much more common [7].

Another important aspect that was to be taken in consideration was the functional outcome following retinal detachment surgery, no statistically significant difference in the final mean BCVA was found between the two groups. These results are in agreement with the results published by Schneider et al., who also reported comparable final BCVA results (without the use of PFCL) to studies where PFCL was used [19].

The mean BCVA on the first postoperative follow-up following the absorption of gas did not differ in eyes with or without displacement evident on FAF. This observation is in line with the studies published by Shiragami et al. and Dell'Omo et al., who both found no correlation between the incidence of retinal displacement and the final BCVA [6,7]

In addition to the final BCVA, symptoms of distortion especially metamorphopsia was detected in 12 eyes of the 14 eyes showing retinal displacement on FAF (85.7\%), 7 of the 8 eyes with retinal displacement in Group (A) (87.5\%) (with the displacement not being macular in the only patient not complaining of metamorphopsia) and in 5 of the 6 eyes ( 4 of whom with displacement involving the macula) with retinal displacement in Group (B) $(83.3 \%)$. This is in accordance with the study published by Lee et al., which reported metamorphopsia in $83 \%$ of patients with macular shift (19/23) [8]. However, of the 19 cases complaining of metamorphopsia only 12 had evidence of displacement on FAF $(63.1 \%)$ which is less than the same study by Lee et al. in which reported that in patients complaining of symptoms of distortion there was evidence of shift in $86 \%$ (19/22) [8]. On the other hand, our results are higher than the results published by Dell'Omo et al., who reported retinal displacement in only $18.1 \%(4 / 22)$ of the patients complaining post-operatively of metamorphopsia, hence we recommend that further research must be put in the post-operative follow-up of patients [7]

Our study was limited by the small sample size, although it does hold the advantage over other studies being randomized in terms of PFCLs use. Also, the group tamponaded with gas without adjuvant PFCLs use and the group tamponaded with gas together with adjuvant PFCL use differed 
with respect to some baseline features like number of detached quadrants, foveal involvement and concomitant involvement of the upper and lower quadrants. Further researches including larger sample size are needed to evaluate causes of retinal displacement and its implications.

\section{Conclusion:}

In conclusion, retinal displacement is common following RRD repair and the use of PFCLs does not seem to affect the incidence of displacement. We found no correlation between the incidence of retinal displacement and the final best corrected visual acuity, but usually it is associated with symptoms of distortion.

\section{Disclosure:}

The author reports no conflicts of interest in this work.

\section{References}

1- ARYA A.V., EMERSON J.W., ENGELBERT M., et al.: Surgical management of pseudophakic retinal detachments: A meta-analysis. Ophthalmology, 113: 1724-1733, 2006.

2- HAGIMURA N., SUTO K., IIDA T., et al.: Optical coherence tomography of the neurosensory retina in rhegmatogenous retinal detachment. Am. J. Ophthalmol., 129: 186-190, 2000.

3- SCHWARTZ S.G. and FLYNN H.W.: Primary retinal detachment: Scleral buckle or pars plana vitrectomy? Curr. Opinion Ophthalmol., 17: 245-250, 2006.

4- YOSHIDA A., OGASAWARA H., JALKH A.E., et al.: Retinal detachment after cataract surgery. Surgical results. Ophthalmology, 99: 460-465, 1992.

5- YU Q., LIU K., SU L., et al.: Perfluorocarbon liquid: Its application in vitreoretinal surgery and related ocular inflammation. Bio. Med. Res. Int., doi:10.1155/2014/ 250323, 2014.

6- SHIRAGAMI C., SHIRAGA F., YAMAJI H., et al.: Unintentional displacement of the retina after standard vitrectomy for rhegmatogenous retinal detachment. Ophthalmology, 117: 86-92, 2010.

7- DELL'OMO R., SCUPOLA A., VIGGIANO D., et al.: Incidence and Factors Influencing Retinal Displacement in Eyes Treated for Rhegmatogenous Retinal Detachment With Vitrectomy and Gas or Silicone Oil. IOVS, 58: 191199, 2017.
8- LEE E., WILLIAMSON T.H., HYSI P., et al.: Macular displacement following rhegmatogenous retinal detachment repair. Br. J. Ophthalmol., 97: 1297-1302, 2013.

9- GARCIA-VALENZUELA E., ITO Y. and ABRAMS G.W. Risk factors for retention of subretinal perfluorocarbon liquid in vitreoretinal surgery. Retina, 24: 746-752, 2004.

10- SCOTT I.U., FLYNN JR. H.W., MURRAY T.G., et al.: Perfluoron Study Group. Outcomes of surgery for retinal detachment associated with proliferative vitreoretinopathy using perfluoro-n-octane: A multicenter study. Am. J. Ophthalmol., 136: 454-463, 2003.

11- KINORI M., MOISSEIEV E., SHOSHANY N., et al.: Comparison of pars plana vi-trectomy with and without scleral buckle for the repair of primary rhegmatogenous retinal detachment. Am. J. Ophthalmol., 152: 291-297, 2011.

12- BARTZ-SCHMIDT K.U., SZURMAN P., WONG D., et al.: Neue Entwicklungen in der chirurgischen Therapie der rhegmatogenen Ablatio retinae. Ophthalmology, 105: 27-36, 2008

13- MARTIíNEZ-CASTILLO V., ESPAX A.B., GARCIíAARUMIí J., et al.: Rate of foveal reattachment. Ophthalmology, 112: 947-948, 2005.

14- BRAZITIKOS P.D., ANDROUDI S., CHRISTEN W.G., et al.: Primary pars plana vitrectomy versus scleral buckle surgery for the treatment of psuedophakic retinal detachment. A Randomized Clinical Trial. Retina., 25: 957-64, 2005.

15- MARTINEZ-CASTILLO V., BOIXADERA A. and GARCIA-ARUMI J.: Pars plana vitrectomy alone with diffuse illumination and vitreous dissection to manage primary retinal detachment with unseen breaks. Arch. Ophthalmol., 127: 1297-1304, 2009.

16- COBOS E., RUBIO M.J., ARIAS L., et al.: Incidence and relation with anatomical and functional variables of postoperative macular displacement in rhegmatogenous retinal detachment. L. Retina., 36: 957-61, 2016.

17- CODENOTTI M., FOGLIATO G., IULIANO L., et al. Influence of intraocular tamponade on unintentional retinal displacement after vitrectomy for rhegmatogenous retinal detachment. Retina., 33: 349-355.

18- PANDYA V.B., HO I.V. and HUNYOR A.P.: Does unintentional macular translocation after retinal detachment repair influence visual outcome? Clin. Exp. Ophthalmol., 40: 88-92, 2012.

19- SCHNEIDER E.W., GERAETS R.L. and JOHNSON M.W.: Pars plana vitrectomy without adjuvant procedures for repair of primary rhegmatogenous retinal detachment. Retina., 32: 213-219, 2012. 


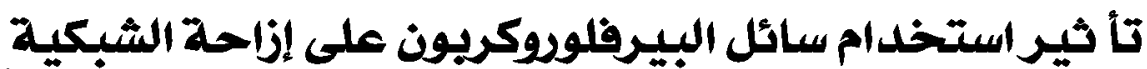

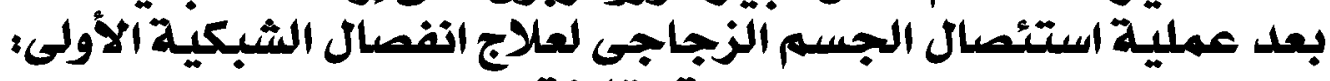 \\ دراسلة مقارنة}

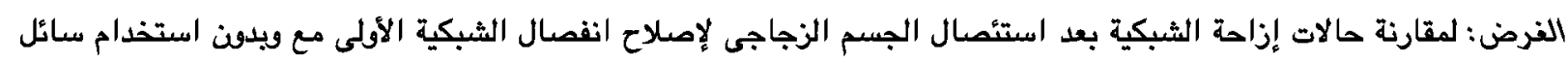

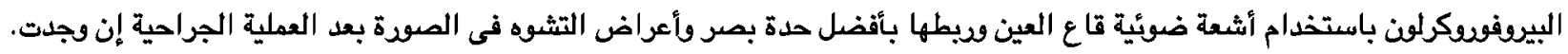

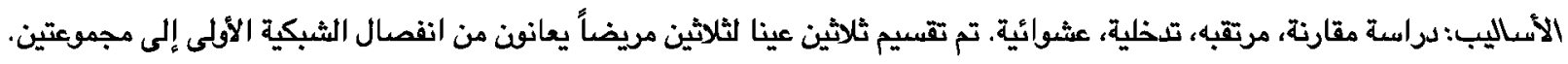

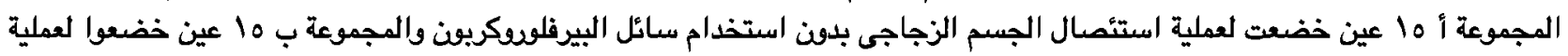

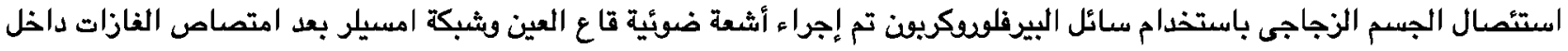

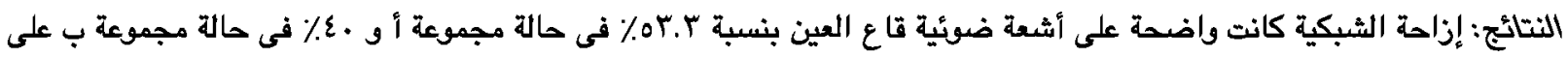

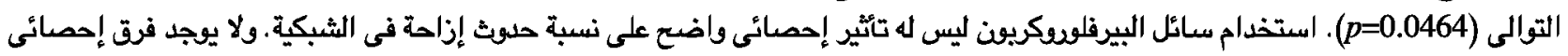

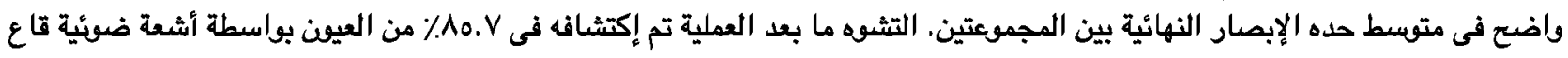
العين.

الخلاصة: إزاحة الشبكية مشكة شائعة بعد عمليات إزالة الجسم الزجاجى لإصلاح الانفصال الشبكى الأولى واستخدام سائل البيروفلوركربيف لا ييدو أنه يؤ ثر على هذه النسبة. 